\title{
COMMISSIONING OF FERMILAB'S ELECTRON COOLING SYSTEM FOR 8-GEV ANTIPROTONS*
}

\author{
S. Nagaitsev ${ }^{\#}$, D. Broemmelsiek, A. Burov, K. Carlson, C. Gattuso, M. Hu, B. Kramper, T. Kroc, \\ J. Leibfritz, L. Prost, S. Pruss, G. Saewert, C.W. Schmidt, A. Shemyakin, M. Sutherland, \\ V. Tupikov, A. Warner, FNAL, Batavia, IL 60134, U.S.A.; S. Seletsky, University of Rochester, \\ Rochester, NY 14627, U.S.A; W. Gai, ANL, Argonne, IL 60439, U.S.A.; G. Kazakevich, Budker \\ INP, Novosibirsk, Russia
}

\begin{abstract}
A 4.3-MeV electron cooling system [1] has been installed at Fermilab in the Recycler antiproton storage ring and is currently being commissioned. The cooling system is designed to assist accumulation of $8.9-\mathrm{GeV} / \mathrm{c}$ antiprotons for the Tevatron collider operations. This paper reports on the progress of the electron beam commissioning effort as well as on detailed plans of demonstrating the cooling of antiprotons.
\end{abstract}

\section{INTRODUCTION}

The Run II Luminosity Upgrade Plan requires the Recycler to play a key role as the repository of large stacks of antiprotons $\left(6 \times 10^{12}\right)$ with the appropriate phase space characteristics to be used in collider stores. In order to maximize the stacking efficiency of the Fermilab antiproton Accumulator, small stacks of antiprotons will be frequently (every 0.5 hour) transferred to the Recycler. In the Recycler, the stacks are initially cooled by stochastic cooling [2] and then stored and cooled by electron cooling until the antiprotons are ready to be used in the Tevatron. The most recent electron cooling status report can be found in Ref. [3].

The Run II Luminosity Upgrade Plan foresees the Recycler fully integrated into collider operations in two major steps. First, the Recycler is commissioned to bring its performance to the level that it is ready to begin the implementation of electron cooling. This milestone was achieved on June 1, 2004. In the second phase, the installation of electron cooling and its commissioning takes place. Electron cooling is expected to be demonstrated by the end of FY05.

This paper outlines the design parameters of the Recycler Electron Cooling System and the commissioning plan that addresses the challenges associated with attaining them. The cooling system commissioning effort consists of four major parts: (1) the Recycler ring commissioning with the electron cooling section, (2) the commissioning of the electron beam line, (3) the demonstration of electron cooling and (4) cooling optimization.

\section{COOLING SYSTEM PARAMETERS}

The electron cooling scenario has been reviewed in Ref. [4]. Under the current scenario the electron cooling system is required to decrease the longitudinal 95\%

*Work supported by the Universities Research Assoc., Inc., under contract DE-AC02-76CH03000 with the U.S. Dept. of Energy.

\#nsergei@fnal.gov emittance of a stored antiproton beam from $100 \mathrm{eV}$-s to $50 \mathrm{eV}$-s in 30 minutes for stacks of up to $6 \times 10^{12}$ particles. This would correspond to providing 36 equally populated bunches with a $1.5-\mathrm{eV}$-s longitudinal emittance per bunch to the Tevatron collider.

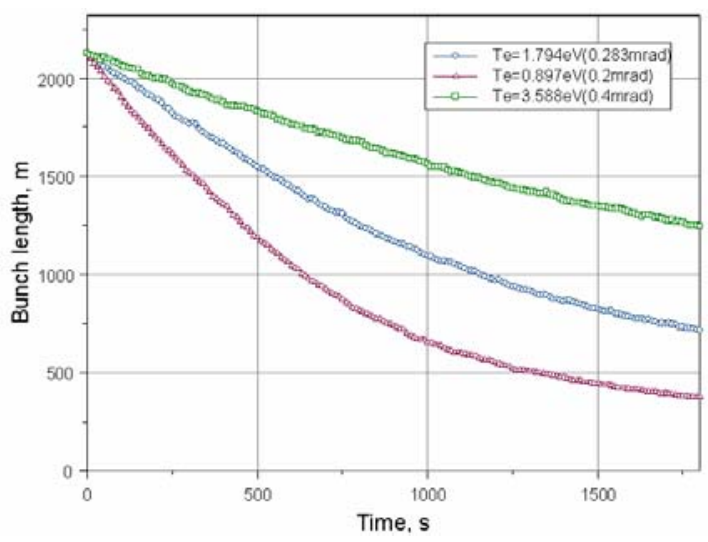

Figure 1: The evolution (simulation) of the antiproton bunch length as a function of time (30 minutes full scale) for various electron beam angular spreads. The initial bunch length corresponds to a $100 \mathrm{eV}$-s longitudinal emittance. The design curve (bottom) indicates that there is a factor of two safety margin in cooling rates.

Figure 1 presents a MOCAC code [5] simulation of the electron cooling process in the Recycler with $6 \times 10^{12}$ antiprotons in a barrier $\mathrm{rf}$ bucket. The three curves correspond to various values of the electron beam rms angular spread in the cooling section. The design value of $0.2 \mathrm{mrad}$ for the rms angular spread is presented by the bottom curve. Other electron beam parameters are presented in Table 1.

Table 1: Electron Cooling System Parameters

\begin{tabular}{|l|c|c|c|}
\hline Parameter & Design & Achieved & Units \\
\hline \hline \multicolumn{3}{|c|}{ Electrostatic Accelerator (at present) } \\
\hline Terminal Voltage & 4.34 & 4.34 & $\mathrm{MV}$ \\
Beam Current & 0.5 & 0.7 & $\mathrm{~A}$ \\
Terminal Voltage & 500 & 500 & $\mathrm{~V}$ \\
Ripple, rms & \multicolumn{3}{|c|}{} \\
\hline \multicolumn{3}{|c|}{ Cooling Section (prototype, 2004) } \\
Length & 20 & 18 & $\mathrm{~m}$ \\
Solenoid Field & $\leq 150$ & 150 & $\mathrm{G}$ \\
Beam Radius & $3-6$ & 6 & $\mathrm{~mm}$ \\
Electron Angular & $\leq 0.2$ & $\leq 0.3$ & $\mathrm{mrad}$ \\
Spread, rms & & & \\
\hline
\end{tabular}


While all of the parameters listed in Table 1 are important for the electron cooling system, attaining three of them, terminal voltage, beam current and the angular spread, concurrently presents a commissioning challenge. The "achieved" column in Table 1 represents the results attained at the R\&D prototype system, which was decommissioned in June, 2004.

The following technical issues have not been fully resolved with the prototype system:

1. Reliability of operations at $4.3 \mathrm{MV}$.

2. Quality of the cooling section magnetic field.

3. High beam losses.

4. Low-frequency electron beam motion.

5. Equipment protection system.

The following section will describe in detail how these issues were addressed prior to commissioning of the cooling system.

\section{COOLING SYSTEM LAYOUT}

Figures 2 and 3 show the Pelletron and the electron cooling system layout.

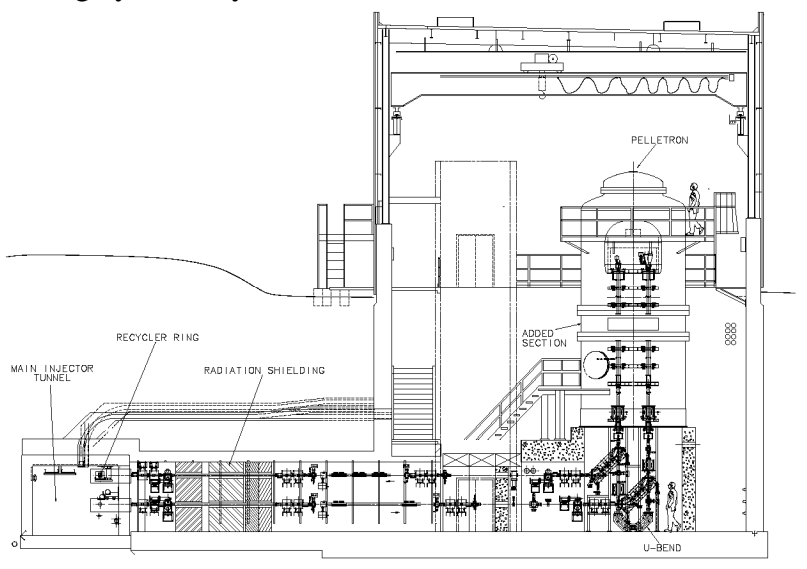

Figure 2: Elevation view showing Pelletron, acceleration and deceleration beam lines, transfer lines passing through connecting enclosure to Recycler ring, and crosssection of Main Injector tunnel which houses the Recycler ring.

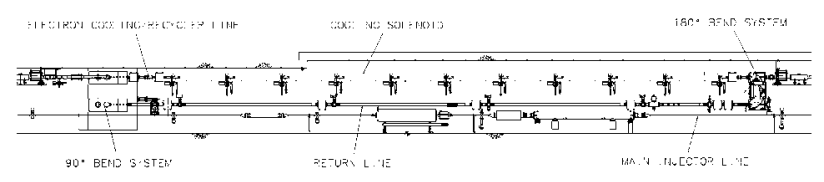

Figure 3: Elevation view of Main Injector tunnel showing the $90^{\circ}$-bend system which injects the electron beam from the transfer line into the Recycler ring, cooling section of Recycler, $180^{\circ}$-bend system which extracts the electron beam from the Recycler, and the return line.

Several modifications have been made to the electron cooling system as compared to the prototype.

1. A sixth acceleration section has been added to the Pelletron (see Fig. 2). This extension has allowed us to increase the stable operation voltage from 3.5 MV to the design value of 4.3 MV. Our experience to date indicates that the Pelletron voltage performance at 4.3 MV is similar or better than that of the prototype system with 5 sections at $3.5 \mathrm{MV}$.

2. A new, fast gun shut-off system will soon be added to make the operations at 4.3 MV more stable.

3. In order to improve the ultimate vacuum level of the acceleration/deceleration tubes, a "dead" section was added to the Pelletron extension to accommodate an additional ion pump. This equipotential space has modified the beam transport optics but thus far has caused no problems with achieving stable DC beam currents.

4. The beam line configuration under the Pelletron has been altered to accommodate both the "U"-shaped beam line used for commissioning in the recirculation experiment, and the vertical bend magnets used to transport the beam to the cooling section (see Fig. 2). This has greatly accelerated the commissioning process by allowing an easy switch over from one mode to the other.

5. A vacuum protection system has been implemented using a series of fast-acting gate valves, cold-cathode gauges, and burst-discs. This system is designed to protect the Recycler ring vacuum system in the event of a catastrophic failure of the vacuum system inside the Pelletron, which could potentially introduce pressurized sulfur hexafluoride gas into the vacuum system.

6. A significant number of diagnostic devices has been added. This includes the addition of a YAG crystal and OTR monitors throughout the beam lines, as well as the addition of a BPM and removable aperture between each of the ten cooling solenoids. The BPMs in the cooling section are capable of monitoring the position of both the antiproton and the electron beams. The position offset between the electron (32 $\mathrm{kHz}$ ) and the antiproton ( $89 \mathrm{kHz}$ ) modes is quite low, which allows the use of the antiproton beam as a reference line.

7. The measured magnetic field quality of the cooling section solenoids is about a factor of two better as compared to the prototype system. This was achieved by improving the magnetic measurement system.

8. A 20-m long, 4-rod beam steering system has been installed in the cooling section to allow for the tilting of the entire electron trajectory.

9. A beam-based procedure for adjusting the cooling section magnetic field quality has been developed.

10. The electron cooling beam lines located in the Main Injector tunnel (Fig. 3) have been magnetically shielded to protect the electron beam from the fields imposed by the ramping of the Main Injector magnetic bus located in near proximity of the electron beamline. Thus far, the electron beam shielding in the cooling section was verified to be effective, while the return beam line requires additional shielding.

11. The equipment-protection permit system has been added and fully commissioned in a U-bend mode. This system is based on a multi-channel $1-\mathrm{kHz}$ 
sampling rate circular buffer, which monitors crucial devices (e.g. loss monitors) and turns the beam OFF when a reading is outside the preset limits. Buffering allows the sequence of events, leading to a trip, to be examined.

12. An explanation and a technical remedy for the high beam losses have been found in test bench measurements.[6]

13. A procedure was developed to measure the electron beam size in the cooling section using a round movable aperture. [7]

The electron cooling assembly was finished in February, 2005 and after some troubleshooting the commissioning began on March 1, 2005.

\section{COMMISSIONING STAGES}

The following commissioning stages were established:

1. Recycler start-up following the cooling system installation.

2. Pelletron commissioning and HV conditioning.

3. U-bend commissioning.

4. Full beam line commissioning.

5. Cooling demonstration.

\section{Recycler start-up}

The Recycler systems and operations had to be adjusted to compensate for the presence of the cooling section. During this commissioning stage the following issues were addressed:

- The Recycler orbit was adjusted and centered through the cooling section BPMs. The machine admittance and the lifetime were measured and found to be acceptable for operations.

- The electron beam dipoles, which bend the beam into and out of the Recycler orbit, were compensated by additional corrector elements immediately upstream and downstream of the cooling section. The solenoid field was determined to be low enough not to cause any additional coupling and was left uncompensated.

- The fast-acting valves used to protect the Recycler vacuum system from a catastrophic failure on the electron cooling side were tested.

These commissioning steps allowed for the electron beam commissioning and the antiproton beam operations to coexist without interfering with one another.

\section{Pelletron commissioning}

After troubleshooting and correcting the controls system, the Pelletron commissioning started with each individual acceleration section being high-voltage conditioned to $1.2 \mathrm{MV}$. It took on average 6 hours to increase the voltage to the point of no vacuum activity. The entire Pelletron acceleration tube was conditioned to 5 MV. The design voltage of $4.3 \mathrm{MV}$ was then established and maintained in a regulation loop with an rms ripple of less than $500 \mathrm{~V}$.

\section{U-bend beamline commissioning}

We allocated a one-month period to establish the fullcurrent beam through the U-bend beamline. In the past prototype tests, we routinely used a microamp-current dc beam together with a rotating-wire position monitor to establish the initial electron trajectory through the beam line. This time, a decision was made to start the commissioning with a pulsed beam instead of a dc beam. This decision was largely based on our experience that a low-current dc beam is the most dangerous for puncturing the vacuum chamber and melting diagnostic instruments. In fact, we did not even install the rotating-wire scanners in the beamline. Low duty-cycle $(2-\mu$ s pulse at $1 \mathrm{~Hz})$ fullcurrent beam pulses are easily detected by our BPM system, thus allowing us to establish the beam optics at a nominal beam current instead of a zero-current pencil beam. This approach was very effective and allowed us to establish dc beam recirculation with low beam losses at the design voltage. The BPM system also operates with a modulated dc beam at currents above about $10 \mathrm{~mA}$. Figure 4 presents the measured beam losses as a function of beam current in a U-bend regime at $4.3 \mathrm{MV}$.

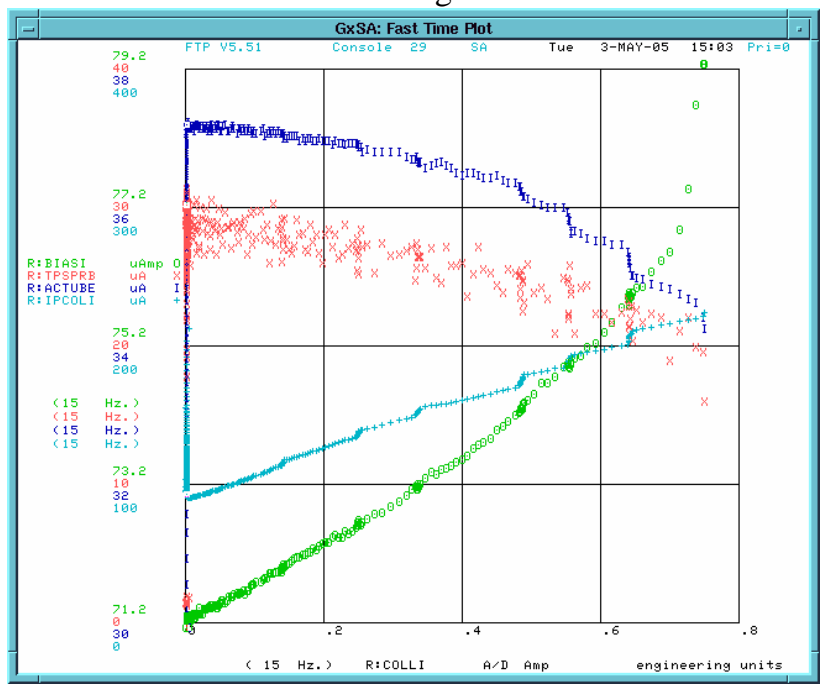

Figure 4: Beam losses (bottom curve, $8-\mu \mathrm{A}$ full scale) as a function of beam current ( $0.8 \mathrm{~A}$ full scale) at $4.3 \mathrm{MV}$.

We presently estimate that in a U-bend regime the total beam loss is $7 \mathrm{ppm}$ at $0.5 \mathrm{~A}$.

The stability of beam operations was demonstrated by running continuously for several hours with a 0.2-A beam. The recirculation interruptions were minor and did not affect the duty cycle.

The beam size measurements, conducted with an OTR monitor, have agreed with the beam envelope model, thus allowing us to adjust the optics file for the full beam line operations.

\section{Full beam line commissioning}

Similarly to the U-bend beamline, the full $80-\mathrm{m}$ long beamline is being first commissioned with a pulsed beam. Although the dc beam of $30 \mathrm{~mA}$ has been already passed through the entire system, the pulsed-beam commissioning continues with several goals in mind: 
- The beam optics needs to be analyzed and adjusted to establish the design beam size and the rms angular spread in the cooling section.

- The electron beam energy needs to be measured and adjusted to match the antiproton energy. The electron beam relativistic factor, $\gamma$, needs to be within $0.3 \%$ of that for the antiproton beam. [8]

By the middle of this summer our plan is to attain a stable beam current of $0.5 \mathrm{~A}$ at the correct energy. Using the BPM system in the dc mode we will adjust the antiproton beam position to be within $0.1 \mathrm{~mm}$ of the electron beam center. Also, we will stabilize the electron beam position by a position feed-back loop.

\section{Cooling process demonstration}

The ultimate goal for the cooling system commissioning effort is to demonstrate the electron cooling of $8.9-\mathrm{GeV} / \mathrm{c}$ antiprotons. There are three major requirements: (1) the beam current needs to be at a $0.5-\mathrm{A}$ level, (2) the rms angular spread of the electron beam needs to be quite low, $0.2 \mathrm{mrad}$ and (3) the energies of the two beams need to be well matched.

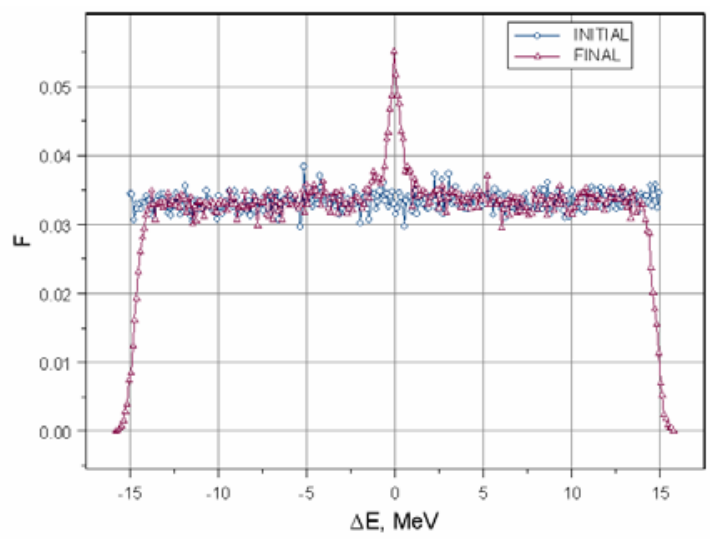

Figure 5: The momentum distribution (arb. units) as a function of antiproton energy deviation (simulation by MOCAC code [5]). The initial distribution is uniform in energy. The final distribution is plotted after 30 minutes.

Since the cooling process is quite slow, we anticipate that the energy matching will be challenging. Our plan is based on two assumptions: (1) the Recycler absolute energy is known to $0.1 \%$ and (2) the Recycler momentum acceptance is greater than $0.3 \%$. Measuring and adjusting the electron absolute energy to $0.3 \%$ would allow us to land the electron beam energy somewhere within the Recycler momentum acceptance. To observe the cooling process we will implement the following procedure. A small $\left(10 \times 10^{10}\right)$ antiproton beam current will be debunched and cooled transversely (by the stochastic cooling system) to a small transverse beam emittance. Using rf noise the momentum spread of this beam will be increased to create a uniform momentum distribution 0.3$0.4 \%$ wide. The cooling process will be observed with the help of a longitudinal beam Schottky-noise monitor, which measures the momentum distribution function. Figure 5 demonstrates a simulation of this process.

The simulation in Fig. 5 was performed with a coasting antiproton beam perfectly matched in energy with the electron beam of $0.1 \mathrm{~A}$ and with $0.5 \mathrm{mrad}$ of rms angular spread. The spike in the distribution, formed by the electron cooling process, increases the distribution function by a factor of $2-$ a value easily detectable by a Schottky-noise spectrum analyzer.

After the cooling demonstration, we will begin a process of optimization to maximize the electron cooling rate. By mid-2006, the cooling system should be fully integrated into the Tevatron collider operations.

\section{CONCLUSIONS}

- The commissioning is on schedule.

- No interference with present Recycler operations is anticipated.

- Cooling demonstration is expected by the end of FY05.

\section{ACKNOWLEDGEMENTS}

The authors are thankful to Fermilab's and Argonne's technical staff for installing the cooling system on schedule. We would like to acknowledge contributions of B. Chase, P. Joireman and J. Crisp for making the BPM system functional; R. Goodwin and L. Carmichael for their help with the protection system; V. Lebedev and D. Johnson for beam optics modeling; and L. Nobrega for designing and installing the fast-acting valve system. We are grateful to P. Zenkevich and A. Bolshakov of ITEP for making their MOCAC simulation code available to us.

\section{REFERENCES}

[1] Prospectus for an electron cooling system for the Recycler. Edited by J.A. MacLachlan, FERMILABTM-2061, Oct 1998.

[2] D. Broemmelsiek, M. Hu and S. Nagaitsev, "Stochastic cooling in barrier buckets at the Fermilab Recycler", EPAC'04, Lucerne, July 2004.

[3] J. Leibfritz et al., "Status of the Fermilab Electron Cooling Project", EPAC'04, Lucerne, July 2004.

[4] A. Burov, "Electron-cooling scenarios at Fermilab", NIM A 532 (2004) 291-297.

[5] P. R. Zenkevich et al., "Modeling of Electron Cooling by Monte Carlo Method", Report at the International Workshop on Cooling and Related Topics, Bad Honnef (Germany), 2001.

[6] L. Prost and A. Shemyakin, "Efficiency of the Fermilab Electron Cooler's Collector", these proceedings (PAC05).

[7] T. Kroc et al., "Electron Beam Size Measurements in a Cooling Solenoid", these proceedings (PAC05).

[8] S. Seletsky and A. Shemyakin, "Beam-Based Calibration of the Electron Energy in the Fermilab Electron Cooler", these proceedings (PAC05). 